\title{
Sub-optimal Power Allocation with Best Relay Algorithm in Multiple Antenna MIMO Relays Networks
}

\author{
Manar Al-Kali \\ School of Computer Science \\ CHINA UNIVERSITY OF GEOSCIENCES \\ No.388 Lumo Road -Wuhan - China
}

\author{
Ali Mohammed \\ Department of Computer Engineering \\ AL-KARKH UNIVERSITY OF SCIENCES \\ Baghdad, Iraq
}

\begin{abstract}
In this paper, the sub-optimal power allocation relay selection (SBS-AF) algorithm in multiple-input multiple-output (MIMO) relay networks was proposed. The energy efficiency and the symbol error rate (SER) in multiple antennas MIMO relay networks is investigated. Amplify and- forward (AF) relay scheme, where $\mathrm{N}$ relay access point(s) occupied with $\mathrm{Q}$ antennas cooperatively forwards packets to the destination is employed. Under the assumption of Rayleigh fading channels and time division multiplexing (TDM), a new exact closedform expressions for the outage probability, SER and the energy efficiency valid for $\mathrm{N}$ Relays and Q antennas was derived. Further asymptotic analysis is done in high SNR regime to characterize the energy efficiency in terms of the diversity order and the array gain. Our scheme was compared with all relay participate (AP-AF) and the recent of best relay selection scheme (S-AF). The results show that our scheme achieves better diversity than the fixed relaying schemes as well maintaining a full diversity of (NQ) +1 . The behavior of the energy efficiency with the relay locations is also discussed in this paper.
\end{abstract}

\section{General Terms}

Energy Efficiency on wireless sensor Networks and Cooperative Networks.

\section{Keywords}

Energy Efficiency, Cooperative Network, Amplify and Forward, MIMO, Power Allocation.

\section{INTRODUCTION}

In wireless distributed networks, packet transmission through wireless medium is quite challenging due to the dynamic channel conditions caused by noise interference, poor liability, and distance expansion. However, cooperative diversity offers an effective solution by exploiting the nearby nodes to assist in the transmission and overcome these drawbacks. It has stood out as an essential concept to the wireless networks and has been discussed in many works. Some of these works have utilized techniques such as distributed beamforming and space-time coding [1]. But these schemes require large feedback overhead and accurate time synchronization. Other studies have considered relay selection algorithms [2]. Nevertheless, drawbacks such as time-space complicities and communication loss have been noted. Multiple-input multiple-output (MIMO) techniques have been proved to be a sold prospective for the wireless networks as well as cooperative communication.

Many works have provided insights into cooperative MIMO network performance. For instance, the ergodic channel capacities for MIMO AF half duplex relay systems are derived by diagonals of the autocorrelation channel matrix from the source to the destination [3]. A novel cooperative detection scheme has been proposed to resolve the complexity of the detection of the relay node [4]. The rate bound with message splitting is utilized in full duplex channel MIMO relay networks [5].

The above-mentioned works illustrate the role of MIMO systems in cooperative communications, because they provide optimal performance in this field. However, like many wireless networks, energy consumption is a major issue in cooperative communications and is a hot topic in recent works. For example, hop distance in combination with the number of nodes was optimized in MIMO and multiple-input single-output (MISO) relay systems to improve their energy consumption [6].

In [7] the Author focus computing the source covariance matrix (or matrices) and the relay transformation matrix to optimize a system performance, however the algorithm is rather complex and applied for only single case when the relay node is an access point. In [8] the author study the structural properties of the optimal power allocation in MIMO-CN with per-node power constraints as it been applied at the source, however schemes require large feedback and knowledge, while [9-10] joint source power allocation and relay beamforming in multi-user multi-relay networks that use single-carrier frequency division multiple access (SC-FDMA) however this approach requires additional data traffic, therefore motivated by [6-10] we propose an amplify-andforward (AF) relay scheme of transmission, where two single antenna nodes communicate via MIMO relay(s) access points occupied with $\mathrm{Q}$ antennas. To obtain maximal achievable received SNR, we assume that the relays is able to perform the rule of maximal ratio combining (MRC) [11] on the received signal as well as the rule of maximal ratio transmitting (MRT) [11] on the forwarded signal. Under independent but not essentially identically distributed (i.n.d) channel conditions, we derive the optimized power allocation formula from which we use to develop our sub-optimal Power allocation algorithm (SBS) to maximize the energy efficiency in our model. Closed-form expressions for the outage probability, SER and the energy efficiency was derived. Then further analyses are provided for high SNR and asymptotic expressions are derived to characterize the energy efficiency in terms of the diversity order and the array gain. Furthermore our result is compared with $\mathrm{AP}-\mathrm{AF}$ and $\mathrm{S}-\mathrm{AF}$, scheme presented in [8]. The simulation results show that the energy efficiency decreases with the diversity order and the location.

\section{SYSTEM MODEL}

We consider MIMO relay network has one source node communicates with the destination node either directly or cooperatively via $N$ MIMO relay access point(s) occupied with $Q$ antennas ( $Q \leq 1$ ), as shown in Figure 1 . 


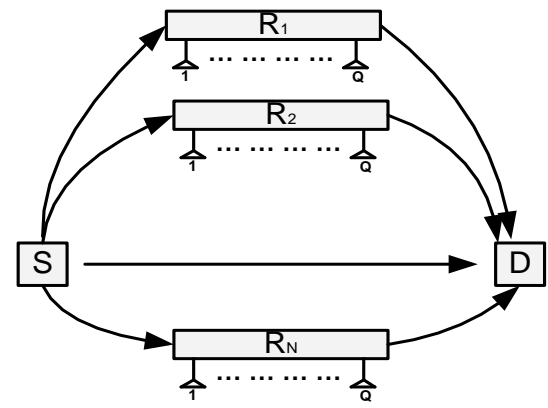

Figure 1. The proposed MIMO network

In this network, i.n.d, half duplex and orthogonal links are used between the nodes, in which the source and the relay transmissions occur in two consecutive time slots.

Assuming that the destination has the knowledge of channel state information (CSI) of all channel hops in the network, and has the ability to utilize the selection diversity combining concept (SC) to identify the channel with the highest instantaneous received SNR between the direct link and the relay links. Furthermore, the destination is also considered to select one "best" relay with the maximal end-to-end SNR to forward the source's signal. In the first time slot the source sends out scaled symbol block with zero mean and unit variance to the other nodes. The received signal at the destination is expressed as:

$$
y_{S D}=H_{S D} P_{s} x+n_{S D}
$$

where $x$ is the symbol block, $H_{S D}$ is the Rayleigh fading coefficient of S - D link, $P_{s}$ is the average transmit power per symbol at the source, and $n_{S D}$ is the additive white Gaussian noise (AWGN) with variance $E\left[n_{S D}\right]=N_{o}$ and $E[$. $]$ is the expected value.

The received signal at the $i_{t h}$ relay is combined with the rule of MRC as in:

$$
y_{S R_{i}}=\varepsilon_{S R_{i}}\left(H_{S R_{i}} P_{s} x+n_{S R_{i}}\right)
$$

where $\varepsilon_{s r}$ is MRC the receive weight vector [11], which is expressed as $\varepsilon_{S R_{i}}=\frac{H_{S R_{i}}^{\dagger}}{\left\|H_{S R_{i}}\right\|^{2}}$ with $\left(^{\dagger}\right)$ and $\|\cdot\|$ denoting the conjugate transpose operation and the vector norm, respectively, $H_{S R}$ is $Q \times 1$ channel vector between the source and relay with Rayleigh fading inputs, and $n_{S R}$ is $Q \times 1$ AWGN with $E\left[n_{S R_{i}} n_{S R_{i}}^{\dagger}\right]=I_{Q} N_{o}$ with $I_{Q}=Q \times Q$ identity matrix.

In the second time slot the $i_{t h}$ relay node amplifies and forwards a scaled copy of (2) to the destination according to the rule of MRT [7]. The received signal at the destination via the relay is given by:

$$
y_{R_{i} D}=\varepsilon_{R_{i} D} G_{i} P_{r} H_{R_{i} D} y_{S R_{i}}+n_{D_{i}}
$$

where $\varepsilon_{R_{i} D}$ is the MRT is transmit weight vector [7], which is given by $\varepsilon_{R_{i} D}=\frac{H_{R_{i} D}^{\dagger}}{\left\|H_{R_{i} D}\right\|^{2}} \quad H_{R D}$ is $1 \times Q$ channel vector between the $i_{t h}$ relay and D with the Rayleigh fading inputs, $P_{R}$ is the average transmit power per symbol at the relay, is the scaling $G_{i}=\frac{1}{\sqrt{P_{S} P_{R_{i}}\left\|H_{R_{i} D}\right\|^{2}+N_{o}}}$ gain in terms of the average SNR of the source/relay channel, and $n_{D}$ is $1 \times Q$ AWGN with $E\left[n_{R D} n_{R D}^{\dagger}\right]=I_{Q} N_{o}$

From (3) the instantaneous received SNR at the destination can be expressed as:

$$
w_{b}=\sum_{i=1}^{N} \frac{G_{i} \cdot P_{S} \frac{\left\|H_{S R_{i}}\right\|^{2}}{N_{o}} \cdot P_{R_{i}} \frac{\left\|H_{R_{i} D}\right\|^{2}}{N_{o} Q}}{P_{S} \frac{\left\|H_{S R_{i}}\right\|^{2}}{N_{o}}+P_{R_{i}} \frac{\left\|H_{R_{i} D}\right\|^{2}}{N_{o} Q}}
$$

where $w_{S R}=P_{S} \frac{\left\|H_{S R}\right\|^{2}}{N_{o}}$ is the instantaneous SNR received from the source to the $i_{\text {th }}$ relay with PDF $f_{w_{S R}}\left(w_{S R}\right)$ and $w_{R D}=P_{R} \frac{\left\|H_{S R_{i}}\right\|^{2}}{N_{o} Q}$ is the instantaneous SNR received from the MIMO relay to the destination with $\mathrm{PDF} f_{w_{R D}}\left(w_{R D}\right)$.

The destination combines the received signals according to the diversity Selection Combining (SC). Finally, the equivalent SNR at the destination is expressed as:

$$
D=\max \left(w_{S D}, w_{b}\right)
$$

where $w_{S D}=P_{s} \frac{\left|H_{S D}\right|^{2}}{N_{o}}$ is the instantaneous SNR from the source to the destination.

\section{PERFORMANCE ANALYSIS}

\subsection{Optimal Power Allocation and SBS Algorithm}

In SBS allocation we simplify (5) by approximating the received SNR at high values, with the help of the inequality of the arithmetic and geometric means ( $\left.\left(\alpha^{-1}+\beta^{-1}\right)^{-1} \leq \frac{1}{2} \sqrt{\alpha \beta}\right)$. We can approximate the expression as follows:

$$
\begin{aligned}
& \gamma(N)=\frac{P_{s}}{N_{o}}\left(\sum_{i=1}^{N} \frac{G_{i} P_{s} P_{k}\left\|H_{S R_{i}}\right\|^{2} \cdot\left\|H_{R_{i} D}\right\|^{2}}{P_{s}\left\|H_{S R_{i}}\right\|^{2}+P_{k}\left\|H_{R_{i} D}\right\|^{2}}\right) \\
& =\frac{1}{N_{o}} \sum_{i=1}^{N}\left[\left(P_{s}\right)^{-1}+\left(G_{i} P_{k}\left\|H_{S R_{i}}\right\|^{2} \cdot\left\|H_{R_{i} D}\right\|^{2}\right)^{-1}\right] \\
& \leq \frac{1}{2 N_{o}} \sum_{i=1}^{N} G_{i} P_{s} P_{k}\left\|H_{S R_{i}}\right\| \cdot\left\|H_{R_{i} D}\right\|
\end{aligned}
$$

Assuming that each relay sends the signal with identical power and the total transmission power of the second phase is denoted as $P_{k}$. For the relay selection, let $T$ denote the number of the selected relays, with SNR output represent as: 


$$
\gamma(T)=\frac{1}{2 N_{o}} \sum_{i=1}^{T} G_{i} P_{s} P_{k}\left\|H_{S R_{i}}\right\| \cdot\left\|H_{R_{i} D}\right\|, i=1, \cdots, T T \leq N
$$

The optimal relay selection set overall relay selection set of $\mathrm{T}$ :

$$
\gamma(T)=\max _{T}(T)
$$

To find the minimum number of $T$ that has maximum received SNR, let the index se of all relays arranged by the rule:

$$
\left\|H_{S R_{i}}\right\| \cdot\left\|H_{R_{i} D}\right\| \geq\left\|H_{S R_{i+1}}\right\| \cdot\left\|H_{R_{i+1} D}\right\|
$$

This arrangement puts the relays with the bad CSI of S-D and D-R links in the bottom of the list, hence initially excluded. Therefore the optimization problem can be expressed:

$$
\max _{P_{s} P_{k}}\left\{\begin{array}{l}
\frac{1}{2 N_{o}}\left(\sum_{i=1}^{T} G_{i} P_{s} P_{k}\left\|H_{S R_{i}}\right\| \cdot\left\|H_{R_{i} D}\right\|\right) \\
\text { s.t } P_{s}+P_{k}=P
\end{array}\right.
$$

By using Lagrangian multiplier, the sub- optimal power allocation is expressed as:

$$
P_{s}^{\prime}=\frac{P}{2}\left(1+\frac{\zeta}{\sqrt{4+\zeta^{2}}}\right) \text { and } P_{k}^{\prime}=\frac{P}{2}\left(1-\frac{\zeta}{\sqrt{4+\zeta^{2}}}\right)
$$

where $\zeta=\frac{\sqrt{T}}{2} \sum_{i=1}^{T} \frac{G_{i}}{\left\|H_{S R_{i}}\right\| \cdot \mid H_{R_{i} D} \|}$

After the relay selection set and power allocation are done, the optimum $N$ of the set $T$ can be found by eliminating the ineffective relay from the set $S$ according to the following condition:

$$
\gamma(T)<\gamma(T-1)
$$

Based on the previous analysis, the SBS algorithm can be illustrated as follows:

1- Let $\mathrm{S}$ is the set of $N$ relays that initially contribute in transmissions

2- Sort the index of all relays according to the rule in (9). Hence the relay with the smaller $\left\|H_{S R_{i}}\right\| \cdot\left\|H_{R_{i} D}\right\|$ is discarded, i.e $\left\|H_{S R_{i}}\right\| \cdot\left\|H_{R_{i} D}\right\|<\gamma_{t h}$

3- Allocate the power between $T$ relays and the source using the SBS power allocation in (11)

4- If $\gamma(T)<\gamma(T-1)$, then exclude $T$ from $S$, else go to step 6 .

5- Let $T=T-1$, if $T \neq 1$, return to step 3,else go to step 6 . 6- End.

\subsection{Outage Probability Analysis}

In this section a new closed-form expression is derived for the cumulative density function (cdf) of $\mathrm{Z}$, which is used to find the outage probability Pout.

At the output of the destination node Pout is defined as $\mathrm{Z}$ falls below a certain threshold, under this condition, the outage probability can be given by:

$$
P_{\text {out }}=\operatorname{Pr}[Z \leq \gamma]=F_{Z}(\gamma)
$$

From the bound in (3.5), the cdf of $\mathrm{Z}$ is expressed as:

$$
F_{Z}(\gamma)=F_{w_{S D}}(\gamma) \cdot F_{w_{b}}(\gamma)
$$

$w_{S D}$ follows an exponential distribution with cdf given by:

$$
F_{w_{o}}(\gamma)=1-e^{-\frac{\gamma}{\bar{w}_{o}}}
$$

where $\bar{w}_{S D}=\frac{P_{S} E\left[\left|H_{S D}\right|\right]^{2}}{N_{o}}$ is the average received SNR from the source to the destination.

Next, the cdf of $w_{b}$ is derived using the following proposition.

The cdf of $w_{q}$ can be expressed as follows:

$$
F_{w_{i}}(\gamma)=\operatorname{Pr}\left[w_{i} \leq \gamma\right]=\int_{\gamma}^{\infty} F_{X}(\gamma+y) f_{Y}(y)
$$

where $F_{w_{S_{i}}}(x)$ is the cdf of $w_{S R}$, which follows a chisquared distribution with $2 \mathrm{Q}$ degree of freedom given by:

$$
F_{w_{S_{i} R}}(x)=1-e^{-\frac{x}{\overline{\bar{w}}_{S R}}} \sum_{q=0}^{Q-1} \frac{x^{q}}{q ! \bar{x}^{q}}
$$

and $f_{w_{R: D}}(y)$ is the pdf of $w_{R D}$, that follows the central chisquared distribution with $2 \mathrm{Q}$ degree as in:

$$
f_{w_{R_{i} D}}(y)=\frac{e^{\frac{y}{\overline{\bar{w}}_{R D}}} y^{Q-1}}{(Q-1) ! \bar{y}^{Q}}
$$

By substituting (3.16) and (3.17) into (3.18) with some algebraic manipulations, the expression is obtained:

$$
F_{W_{i}}(\gamma)=1-\frac{e^{-\frac{\gamma}{\bar{w}_{R_{i} D}}}}{(Q-1) ! \bar{w}_{R_{i} D}^{Q}} \sum_{q=0}^{Q-1} \frac{1}{q ! \bar{w}_{S_{i} R}^{q}} \sum_{n=0}^{q}\left(\begin{array}{l}
q \\
n
\end{array}\right) \gamma^{q-n} \int_{\gamma}^{\infty} y^{Q-n-1} e^{-\frac{\gamma}{\overline{\bar{w}_{i} R}}} \cdot d y
$$

The integral in appendix B at (B.4) is solved with the identity $[12,3.381 .3]$ :

$$
\int_{t}^{\infty} t^{v-1} e^{-u t} \cdot d t=u^{-v} \Gamma(v, u t)
$$

yields to the expression in to the cdf at the $i_{\text {th }}$ relay node occupied with Q antennas is given by:

$$
\begin{gathered}
F_{w_{i}}(\gamma)=1-\frac{1}{(Q-1) ! \bar{w}_{R_{i} D}^{Q}} \sum_{q=0}^{Q-1} \frac{1}{q ! \bar{w}_{S R_{i}}^{q}} \sum_{z=0}^{q}\left(\begin{array}{l}
q \\
z
\end{array}\right)\left(\frac{1}{\bar{w}_{i_{q}}}\right)^{Q+n} \\
\times \gamma^{q-n} e^{\frac{\gamma}{\overline{\bar{w}}_{S R}}} \Gamma\left(Q+z, \frac{\gamma}{\bar{w}_{i_{q}}}\right)
\end{gathered}
$$

where $\bar{w}_{S R_{i}}=\frac{P_{S} E\left[\left\|H_{S R_{i}}\right\|^{2}\right]}{N_{o}} \forall q \in[1, \ldots, Q]$ is the average SNR from the source to the MIMO relay, $\bar{w}_{R_{i} D}=\frac{P_{R_{i}} E\left[\left\|H_{R_{i} D}\right\|^{2}\right]}{N_{o} Q} \forall q \in[Q, \ldots, 1]$ is the average SNR from the MIMO relay to the destination, $\bar{w}_{i_{q}}=\frac{Q \bar{w}_{S R_{i}} \cdot \bar{w}_{R_{i} D}}{\bar{w}_{S R_{i}}+\bar{w}_{R_{i} D}}$ is the end to end average SNR over the path through the relay. 
Since $w_{b}=\arg \max _{i} w_{i}$, then the cdf of the best selected relay can be expressed as:

$F_{w_{b}}(\gamma)=\left[F_{w_{i}}(\gamma)\right]^{N}=\sum_{n=0}^{N-1}\left(\begin{array}{l}N-1 \\ n\end{array}\right) \times(-1)^{N-n-1}\left[F_{w_{i}}(\gamma)\right]^{N-n-1}$

By using the multinomial theorem, the new exact expression of the cdf of the best relay Selected in the network can be expressed in (23):

$F_{w_{b}}(\gamma)=\sum_{n=0}^{N-1}\left(\begin{array}{l}N-1 \\ n\end{array}\right) \times(-1)^{N-n-1} \frac{1}{(Q-1) ! \bar{w}_{R, D}^{Q}} \sum_{|\mathrm{g}|=N-n-1}(N-n-1) ! \prod_{q=0}^{Q-1} \frac{1}{\left(q ! \bar{w}_{S R}^{q}\right)^{g_{q}} g_{q} !}$

$\sum_{k=0}^{\mu}\left(\begin{array}{l}\mu \\ k\end{array}\right)\left(\frac{N-n-1}{\bar{w}_{i_{q}}}\right)^{Q+k} \times \gamma^{\mu-k} e^{\frac{\gamma(N-n-1)}{\bar{w}_{S R}}} \Gamma\left(Q+k, \frac{\gamma(N-n-1)}{\bar{w}_{i_{q}}}\right)$

where $\mathbf{g}=\left(g_{0}, g_{1} \mathrm{~K}, g_{q}, \mathrm{~K} g_{Q-1}\right)$ is the Q-dimension vector of non-negative integers with the length definition of $|\mathrm{g}|=\sum_{q=0}^{Q-1} g_{q}$, the second summation term means that sum is taken all g of length $y-t-1, \mu=\sum_{q=0}^{Q-1} q g_{q}$.

By substituting (15), (23) into (13) and applying the identity in (14) with some algebraic manipulation, the new closedform expression for the end to end outage probability at the destination (Pout) is presented in (24), which is valid for an arbitrary $\mathrm{Q}$ of antennas at the relay.

\subsection{Symbol Error Rate Analysis}

In this section, closed-form expression for SER is derived using the cdf result in the previous section. The resultant expression is valid for all general modulation forms that have the conditional error probability (CEP) of the form $Q_{e}(\sqrt{2 \beta \gamma})$

where $Q_{e}($.$) is the Gaussian Q-function and \beta=\sin ^{2}\left(\frac{\pi}{M}\right)$ for M-ary phase shift keying (MPSK).

The most common approach to evaluate the SER is by integrating the CEP over the probability density function (pdf) of the received SNR [13]:

$$
P_{e}=E[Q(\sqrt{2 \beta \gamma})]=\frac{1}{\sqrt{\pi}} \int_{0}^{\infty}\left[\int_{\sqrt{\beta \gamma}}^{\infty} \frac{e^{-\beta \gamma}}{\sqrt{\gamma}} f_{Z}(\gamma) \cdot d \gamma\right]
$$

However, this work adopt the approach developed in [14-15], which allows us to express the SER directly using the cdf of the received SNR as follows:

$$
P_{e}=\frac{\sqrt{\beta}}{2 \sqrt{\pi}} \int_{0}^{\infty} \frac{e^{-\beta \gamma}}{\sqrt{\gamma}} F_{Z}(\gamma) \cdot d \gamma
$$

Substituting (3.24) into (3.26) with some algebraic manipulation, yields:(27) solving the integral by applying the identity [12, 4.55.1]:

$$
\int_{0}^{\infty} t^{\mu-1} e^{-p t} \Gamma(r, c t) \cdot d t=\frac{c^{r} \Gamma(\mu+r)}{\mu(c+p)^{\mu+r}}{ }_{2} F_{1}\left(1, \mu+r ; \mu+1 ; \frac{p}{c+p}\right)(28)
$$

where ${ }_{2} F_{1}(a, b ; c ; d)$ is the hypergeometric function defined in [16]. Finally the exact SER can be expressed as (29).

The result in (29) is the new closed-form expression for the SER of the MIMO relaying, where

$$
\begin{aligned}
& \alpha=Q+\mu+\frac{1}{2}, \quad t=\mu-k+\frac{1}{2}, \rho=Q-k+\frac{1}{2}, \\
& c_{1}=(N-n-1)\left(\frac{\beta}{N-n-1}+\frac{1}{\bar{w}_{S D}}+\frac{1}{\bar{w}_{S R}}\right) \quad c_{2}=(N-n-1)\left(\frac{\beta}{N-n-1}+\frac{1}{\bar{w}_{S R}}\right) \\
& \delta_{1}=(N-n-1)\left(\frac{\beta}{N-n-1}+\frac{1}{\bar{w}_{i_{q}}}+\frac{1}{\bar{w}_{S R}}\right) \quad \delta_{2}=(N-n-1)\left(\frac{\beta}{N-n-1}+\frac{1}{\bar{w}_{i_{q}}}+\frac{1}{\bar{w}_{S D}}+\frac{1}{\bar{w}_{S R}}\right)
\end{aligned}
$$

\subsection{Energy Efficiency}

Using the SER expression derived in before to derive the energy efficiency according to [13]:

$$
\eta=\frac{L\left(1-P_{e}\right)}{E}
$$

$L$ is the length of packet, $E$ is the total energy consumption and $P_{e}$ represents the Symbol error probability at the destination.

$E$ can be calculated according to [13] as:

$$
E=\frac{L_{p} P}{R_{b}}
$$

where $P$ is the total consumed power, $L_{p}$ is the payload packet length and $R_{b}$ as the bit rate.

The total power in AF MIMO relaying can be expressed as:

$$
P_{\text {total }}=P_{s}(1+\lambda) \sum_{i=1}^{N}\left(p_{c t}+\sum_{q=1}^{Q} p_{c r}\right)+\sum_{i=1}^{N} \frac{P_{r_{i}}}{Q}(1+\lambda)\left(\sum_{q=1}^{Q} p_{c t}+\sum_{q=1}^{Q+1} p_{c r}\right)(32)
$$

where, $\lambda$ is the loss factor of the power amplifier, that lies in the range of $(0 \leq \lambda \leq 1)$, while (and $\left.P_{c r}\right)$ are the power consumption of the circuit block of the transmitter and the receiver respectively .

Substituting (32) into (31), the expression for the energy consumption as:

$$
E_{\text {Total }}=\frac{L_{p} P_{s}(1+\lambda)\left(p_{c t}+\sum_{i=1}^{N} \sum_{q=1}^{Q} p_{c r}\right)+\sum_{i=1}^{N} \frac{P_{r i}}{Q}(1+\lambda)\left(\sum_{q=1}^{Q} p_{c t}+\sum_{q=1}^{Q+1} p_{c r}\right)}{R_{b i t}}
$$

Finally, applying (29) and (33) into (30) the closed form expression for the energy efficiency for MIMO relay networks, expressed in (3.34):

Our expressions is a straight forward and easy to compute that composes from finite summations of standard hypergeometric functions, which are easy to simulate in software such as Mathematica or Matlab

\subsection{High SNR Evaluation}

The closed-form expressions in the previous sections can be further analyzed for the high SNR regime by adopting the approach in [16] that for the uncoded systems, the outage probability and SER are approximated by a single polynomial term for $\gamma \rightarrow \infty$

$$
\begin{gathered}
P_{\text {out }}^{\infty}=\frac{a}{t+1}\left(\frac{\gamma}{\bar{\gamma}}\right)^{t+1}+o\left(\gamma^{-t+1}\right) \\
P_{e}^{\infty}=\frac{2^{t} a \Gamma\left(t+\frac{3}{2}\right)}{\sqrt{\pi}(t+1)}(\beta \bar{\gamma})^{t+1}+o\left(\bar{\gamma}^{-t+1}\right)
\end{gathered}
$$




$$
\begin{gathered}
P_{\text {out }}=\sum_{n=0}^{N-1}\left(\begin{array}{l}
N-1 \\
n
\end{array}\right) \times(-1)^{N-n-1} \frac{1}{(Q-1) ! \bar{w}_{R_{i} D}^{Q}} \sum_{|\mathbf{g}|=N-n-1}(N-n-1) ! \prod_{q=0}^{Q-1} \frac{1}{\left(q ! \bar{w}_{S R_{i}}^{q}\right)^{g_{q}} g_{q} !} \sum_{k=0}^{\mu}\left(\begin{array}{l}
\mu \\
k
\end{array}\right)\left(\frac{N-n-1}{\bar{w}_{i_{q}}}\right)^{Q+k} \gamma^{\mu-k} e^{-\gamma\left(\frac{(N-n-1)}{\bar{w}_{S R}}\right)} \Gamma\left(Q+k, \frac{\gamma(N-n-1)}{\bar{w}_{i_{q}}}\right) \\
-\sum_{n=0}^{N-1}\left(\begin{array}{l}
N-1 \\
n
\end{array}\right) \times(-1)^{N-n-1} \frac{1}{(Q-1) ! \bar{w}_{R_{i} D}^{Q} D} \sum_{|\mathbf{g}|=N-n-1}(N-n-1) ! \prod_{q=0}^{Q-1} \frac{1}{\left(q ! \bar{w}_{S R_{i}}^{q}\right)^{g_{q}} g_{q} ! ! k=0} \sum_{k}^{\mu}\left(\begin{array}{l}
\mu \\
k
\end{array}\right)\left(\frac{N-n-1}{\bar{w}_{i_{q}}}\right)^{Q+k} \times \gamma^{\mu-k} e^{-\gamma\left(\frac{(N-n-1)}{\bar{w}_{S R}}+\frac{1}{\bar{w}_{S D}}\right)} \Gamma\left(Q+k, \frac{\gamma(N-n-1)}{\bar{w}_{i_{q}}}\right)(24)
\end{gathered}
$$

$$
\begin{aligned}
& P_{e}=\sum_{n=0}^{N-1}\left(\begin{array}{l}
N-1 \\
n
\end{array}\right) \times(-1)^{N-n-1} \frac{1}{(Q-1) ! \bar{w}_{R_{i} D}^{Q}} \sum_{|\mathrm{g}|=N-n-1}(N-n-1) ! \prod_{q=0}^{Q-1} \frac{1}{\left(q ! \bar{w}_{S R_{i}}^{q}\right)^{g_{q}} g_{q} !} \sum_{k=0}^{\mu}\left(\begin{array}{c}
\mu \\
k
\end{array}\right)\left(\frac{N-n-1}{\bar{w}_{i_{q}}}\right)^{Q+k} \\
& \times\left(\int_{0}^{\infty} \gamma^{\mu-k} \Gamma\left(Q+k, \frac{\gamma(N-n-1)}{\bar{w}_{i_{q}}}\right)\left(e^{-\gamma\left(\beta+\frac{1}{\overline{\bar{w}}_{S R}}\right)}-e^{-\gamma\left(\beta+\frac{1}{\overline{\bar{w}}_{S D}}+\frac{1}{\overline{\bar{w}}_{S R}}\right)}\right) \cdot d \gamma\right) \\
& P_{e}=\sum_{n=0}^{N-1}\left(\begin{array}{l}
N-1 \\
n
\end{array}\right) \times(-1)^{N-n-1} \frac{1}{(Q-1) ! \bar{w}_{R_{i} D}^{Q}} \sum_{|\mathrm{g}|=N-n-1}(N-n-1) ! \prod_{q=0}^{Q-1} \frac{1}{\left(q ! \bar{w}_{S R_{i}}^{q}\right)^{g_{q}} g_{q} !} \sum_{k=0}^{\mu}\left(\begin{array}{c}
\mu \\
k
\end{array}\right)\left(\frac{N-n-1}{\bar{w}_{i_{q}}}\right)^{2(Q+k)} \\
& \times\left(\frac{\Gamma(\alpha)}{t\left(\delta_{1}\right)^{\alpha}}{ }_{2} F_{1}(1, \alpha ; \rho+1) ; \frac{c_{1}}{\delta_{1}+\beta+\frac{1}{\bar{w}_{S R}}}-\frac{\Gamma(\alpha)}{t\left(\delta_{2}\right)^{\alpha}}{ }_{2} F_{1}(1, \alpha ; \rho+1) ; \frac{c_{2}}{\delta_{2}+\beta+\frac{1}{\bar{w}_{S R}}+\frac{1}{\bar{w}_{S D}}}\right) \\
& \eta=\frac{L R_{b i t}}{L_{p} \sum_{i=1}^{N} P_{s}(1+\lambda)\left[p_{c t}+\sum_{q=1}^{Q} p_{c r}\right]+\sum_{i=1}^{N} \frac{P_{r_{i}}}{Q}(1+\lambda)\left[\sum_{q=1}^{Q} p_{c t}+\sum_{q=1}^{Q+1} p_{c r}\right]}\left[\sum_{n=0}^{N-1}\left(\begin{array}{l}
N-1 \\
n
\end{array}\right) \times(-1)^{N-n-1} \frac{1}{(Q-1) ! \bar{w}_{R_{i} D}^{Q}|\lg |=N-n-1} \sum_{(N-n-1) ! \prod_{q=0}^{Q-1}} \frac{1}{\left(q ! \bar{w}_{S R_{i}}^{q}\right)^{g_{q}} g_{q} !}\right. \\
& \left.\times \sum_{k=0}^{\mu}\left(\begin{array}{l}
\mu \\
k
\end{array}\right)\left(\frac{N-n-1}{\bar{w}_{i_{q}}}\right)^{2(Q+k)}\left(\frac{\Gamma(\alpha)}{t\left(\delta_{1}\right)^{\alpha}}{ }_{2} F_{1}(1, \alpha ; \rho+1) ; \frac{c_{1}}{\delta_{1}+\beta+\frac{1}{\bar{w}_{S R}}}-\frac{\Gamma(\alpha)}{t\left(\delta_{2}\right)^{\alpha}}{ }_{2} F_{1}(1, \alpha ; \rho+1) ; \frac{c_{2}}{\delta_{2}+\beta+\frac{1}{\bar{w}_{S R}}+\frac{1}{\bar{w}_{S D}}}\right)\right]
\end{aligned}
$$

Applying the identity in $[8,8.339 .2]$, SER in (3.36) can be further simplified for $\mathrm{t}$ is a positive integer:

$$
P_{e}^{\infty}=\frac{a t(2 t+1) !}{2^{t+1}(t+1) t !}(\beta \bar{\gamma})^{t+1}+o\left(\bar{\gamma}^{t+1}\right)
$$

The asymptotic outage probability is derived using the following proposition

Proposition 3.1: For high SNR regime the asymptotic outage probability in (33) is derived from the exact expression in (19) with the help of the generalized power series expansion and the series representation of the incomplete gamma function. While deriving the asymptotic outage probability, the received SNRs of the cooperative path are re-expressed as $\bar{w}_{S R}=\tau_{S R} \bar{w}_{S D}, \bar{w}_{R D}=\tau_{R D} \bar{w}_{S D} / Q_{\text {and }} Q \leq 1$ and $N \leq 1$.

$P_{\text {out }}^{\infty}=\left(\frac{\gamma}{\bar{w}_{S D}}\right)^{L+1}\left(\frac{L}{L ! \bar{w}_{S D} \tau_{R D}}+\frac{1}{(L-1) ! \bar{w}_{S D} \tau_{S R}}\right)+o\left(\bar{w}_{S D}{ }^{(L+1)}\right)$

Proof: see appendix A

Next, the asymptotic expression of SER is derived by comparing the result in (38) with (24). Substituting the corresponding values of $a$ and $t$ into (37), the asymptotic expression of SER as in:

$$
\begin{gathered}
P_{e}^{\infty}=\frac{L(2 L+1) !}{2^{L+1}(L+1) L !}\left(\frac{\gamma}{\bar{w}_{S D}}\right)^{L+1}\left(\frac{L}{L ! \bar{w}_{S D} \tau_{R D}}+\frac{1}{(L-1) \bar{w}_{S D} \tau_{S_{i} R}}\right) \\
\times\left(\beta \bar{w}_{S D}\right)^{-L+1}+o\left(\bar{w}_{S D}^{-(L+1)}\right)
\end{gathered}
$$

According to [16], the SER in high regime can be re-written:

$$
P_{e}^{\infty}=\left(\zeta_{a}\right)^{-\xi_{d}}+o\left(\bar{\gamma}^{\zeta_{d}}\right)
$$

where $\xi_{d}$ is the diversity order and $\xi_{a}$ denotes as the array gain. As it seen, the asymptotic SER in (3.39) achieves maximum diversity order of $\xi_{d}=L+1$. The corresponding array gain is given by:

$$
\zeta_{a}=\frac{L(2 L+1) !}{2^{L+1}(L+1) L !}\left(\frac{\gamma}{\bar{w}_{S D}}\right)^{L+1}\left(\frac{L}{L ! \bar{w}_{S D} \tau_{R_{i} D}}+\frac{1}{(L-1) ! \bar{w}_{S D} \tau_{S R}}\right)
$$

From (41), $\xi_{a}$ increases with respect to $\tau_{S R_{i}}=\bar{w}_{S R} / \bar{w}_{S D}$ and $\tau_{R_{i} D}=Q \bar{w}_{R_{i} D} / \bar{w}_{S D}$. Substituting (41) into (31) with some algebraic maculation the asymptotic expression of the energy efficiency in term of array gain as follows:

$$
\eta=\frac{L R_{b i t}}{L_{p}}\left(\frac{1}{1+\left(\bar{w}_{S D} \zeta_{a}\right)^{-\xi_{d}}}\right)
$$


As in (42) shows that $\eta$ decreases with respect to the increment of $\xi_{a}$ and $\xi_{d}$.

\section{NUMERICAL RESULT}

In this section, numerical results are presented to compare our scheme with fixed relaying schemes and to demonstrate the impacts of $Q$ and $N$ on the average received SNRs, the outage probability, SER and the energy efficiency. Our results are evaluated by Mathematica 5.2, where Monte Carlo simulations are performed in each case to compare and to investigate the reliability of our analytical expressions. Assuming the channel are generated with normalized noise gain and variance conditions, i.e.

$$
\left(N_{o}=E\left[\left|H_{S D}\right|^{2}\right]=E\left[\left\|H_{S R}\right\|^{2}\right]=E\left[\left\|H_{R D}\right\|^{2}\right]=1\right) .
$$

Moreover, the specification of mica2mote [19] are adopted in our simulations, i.e.

$$
\begin{aligned}
& \left(p_{c t}=10^{-4}, p_{c r}=5 \times 10^{-5}, L=80, L_{p}=68 \text { and } \mathrm{R}_{b}=10^{5}\right) \text { and } \\
& P_{S_{i}}=\tau_{S_{i} R} P \text { and } P_{R_{i}}=\tau_{R_{i} D} P \quad \text { with } \tau_{S_{i} R} \text { and } \tau_{R_{i} D} \text { are real }
\end{aligned}
$$

positive numbers of the range $(\tau>0)$ and $P$ as the total power in the system. Our results are compared with AP-AF and (S-AF) a selection scheme in presented in [8]. Similar conclusion can be obtained from both the outage probability and SER; hence only results for SER and the energy efficiency are presented.

The energy efficiency performance of SBS-AF, AP-AF and S$\mathrm{AF}$ are plotted in Figure 2. As it is clear from the figure the enhancement of the energy efficiency in SBS-AF is about $6.21 \times 10^{3} \mathrm{bit} / \mathrm{J}$ over $\mathrm{S}-\mathrm{AF}$ and $1.461 \times 10^{4} \mathrm{bit} / \mathrm{J}$ over AP$\mathrm{AF}$ at $P_{e}=10^{-2}$.

From the obtained result it is clear that the power allocation in SBS-AF scheme can optimize the energy consumed in both the transmission and in the forwarding respectively and thus improving the energy efficiency in a better way than the fixed relaying schemes.

Figure 3. plots the exact and asymptotic SER for different numbers of $Q$ at the relays with $N=2$ and BPSK modulation. Consider the balanced hop case, i.e. $\tau_{S R}=\tau_{R D}$ and $\bar{w}_{S D}=\bar{w}_{S R}=Q \bar{w}_{R D}$. The figure shows there are significant variations in the slope of the curves which is the reflection of the diversity order. When $P_{e}=10^{-2}$ the $Q=3$ antennas system achieves about $6: 1 \mathrm{~dB}$ array gain than the $Q=2$ antennas system, which confirms our conclusion that the diversity order increase with increasing $Q$ according to $\zeta_{d}=2 Q+1$

Observing in the figure that the exact and the asymptotic expressions converge to the same value in high SNR regime. The figure also shows a comparison between Monte Carlo simulations results and the analytical expressions, which reveals the correctness of results with tolerance less than $1 \%$.

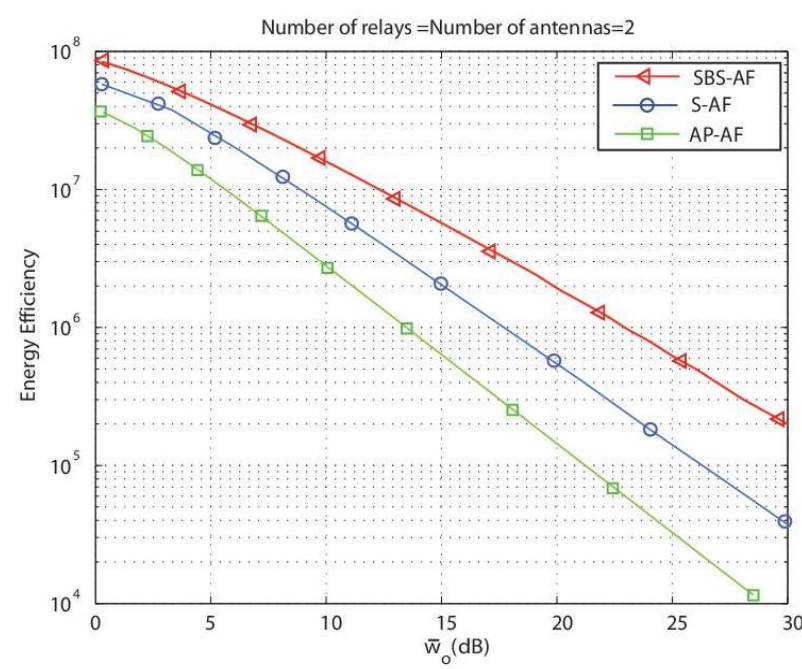

Figure 2.Comparison of energy efficiency for SBS-AF, S$A F$ and AP-AF versus wSD for the balanced hops case with $\mathrm{N}=2, \mathrm{Q}=2$ and $\mathrm{BPSK}$.

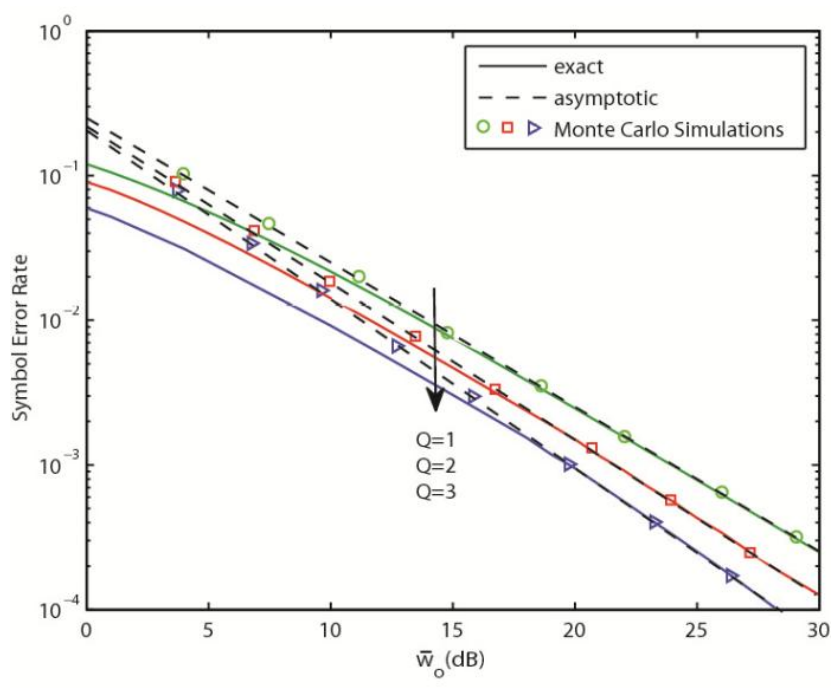

Figure.3. Exact, asymptotic and simulations of SER for SBS-AF versus wSD for the balanced hops case with $\mathrm{N}=2$ and BPSK modulation

Figure 4. plots the performance of SER for unbalanced hop case, in which by considering two locations for the relay access point to be presented.

Case A: is when the relay is closer to the source, i.e. $\tau_{S R}=2$ and $\tau_{R D}=1$, therefore $\bar{w}_{S R}=2 \bar{w}_{S D}$ and $\bar{w}_{R D}=\bar{w}_{S D} / Q$

Case B: is when the relay is closer to the destination, i.e. $\tau_{R D}=1$ and $\tau_{S R}=2$, so $\bar{w}_{S R}=\bar{w}_{S D}$ and $\bar{w}_{R D}=2 \bar{w}_{S D} / Q$.

The result in Fig.4. Shows that the diversity order of $(\mathrm{Q}+1)$ is achieved by both cases. In low SNR regime, for example $P_{e}=10^{-2}$ Case A and case B achieves $1: 2 \mathrm{~dB}$ and 0:6 dB array gain compared to the balanced hops case. However in high SNR regime the gap between the two cases becomes larger, as case A converge with the balanced hops case to the same value. 


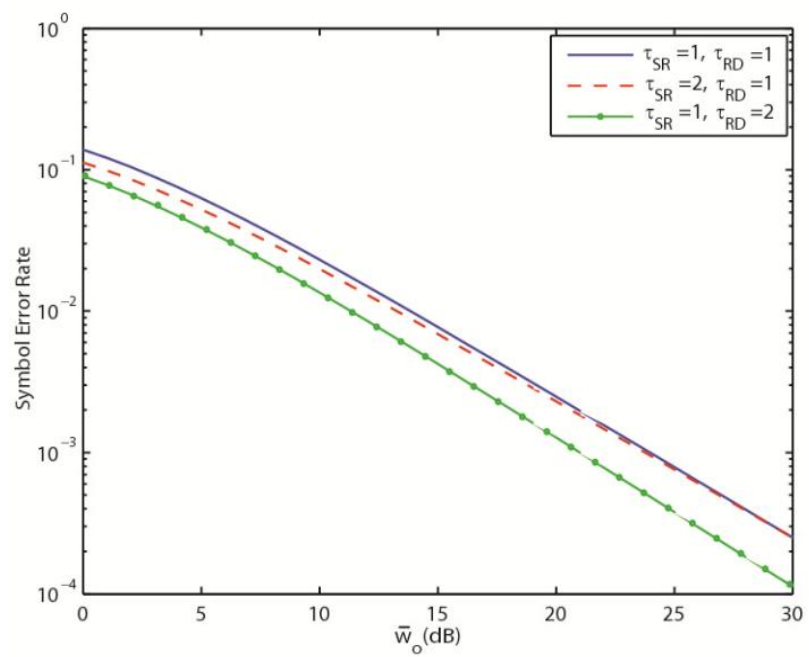

Figure 4. Exact SER versus wSD for the unbalanced hops with $\mathrm{N}=2$ and BPSK modulation.

Figure 5. plots the exact and asymptotic energy efficiency for different numbers of $Q$ at the relay with BPSK modulation. Under the balanced hops conditions, it is clear that the energy efficiency achievement decreases with the increment in number of antennas in the relay according to $\xi_{d}=2 Q+1$. For example, when $\bar{w}_{S D}=6 \mathrm{~dB}$, the energy efficiency drops about $4 \times 106 \mathrm{bit} / \mathrm{J}$ by increasing $\mathrm{Q}$ from 2 to 3 . This confirms our conclusion that the system attains minimum energy efficiency when it achieves a maximum diversity order of $\mathrm{L}+$ 1. The figure also shows that in high SNR regime, the exact and the asymptotic expressions converge to the same value.

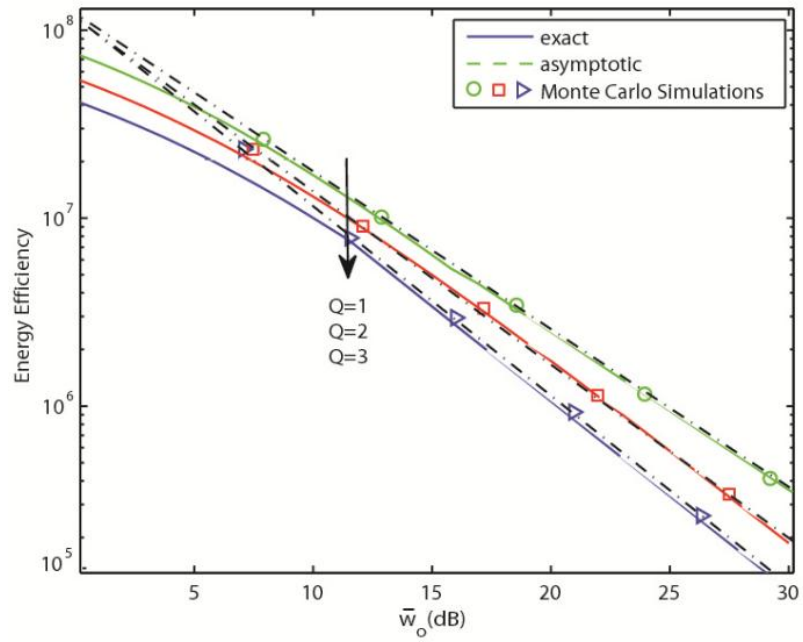

Figure 5. Exact, asymptotic and simulations of energy efficiency versus wSD for the balanced hops case with $\mathrm{N}=2$ and BPSK modulation

Figure 6. plots the energy efficiency performance for the unbalanced hops. The results shows that both case A and case $B$ attain close energy efficiency performance in low SNR regime, for example at, case $\mathrm{A}$ and case $\mathrm{B}$ provide $3.0952 \times 107 \mathrm{bit} / \mathrm{J}$ an $3.1074 \times 107 \mathrm{bit} / \mathrm{J}$ respectively

However in high SNR regime the gap between their curves become larger, as case A converge with the balanced case to the same value. When $\bar{w}_{S D}=18 \mathrm{~dB}$, case B provides $8.9 \times$ $104 \mathrm{bit} / \mathrm{J}$ compared to the other two cases. This conclusion can help to design practical systems with optimal relay location to minimize the SER and maximize the energy efficiency.

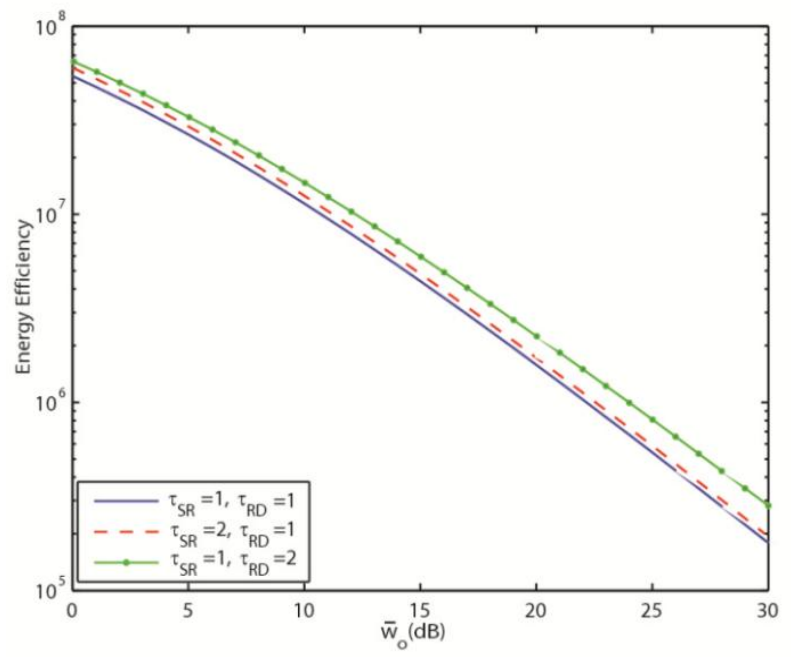

Figure 6. Exact energy efficiency from versus wSD for the unbalanced hops with $\mathrm{N}=2$ and BPSK modulation

\section{CONCLUSION}

In this paper, the energy efficiency and SER of MIMO cooperative relay networks were investigated. Under Rayleigh fading conditions, an AF relay network, where two nodes communicate with each other either directly or cooperatively via $N$ access point relays node occupied with $Q$ antennas is employed. TDM multiplexing was utilized in the relay to ensure consecutive transmissions through the network. Specifically, new selection scheme SBS-AF and analyzed the impact of number relays and number of antennas and the average received SNR on the energy efficiency, the outage probability and SER in i.n.d channel characteristics is proposed. The analysis was used to derive new closed-form expressions of the outage probability SER and $\eta$ valid for $N$ numbers of relays. For high SNR regime new asymptotic expressions to obtain the diversity order and array gain is obtained, which are important parameters for the design of an energy efficient wireless distributed networks. From the result, it is clear that the energy efficiency decreases with number of relays and antennas according to $L+1$. Finally, the impact of the relay location on the energy efficiency and SER is studied. Our results revealed that the SBS-AF scheme achieves the best energy efficiency than the other fixed.

\section{APPENDIX A}

Using the identity:

$$
e^{t} \sum_{n=0}^{N-1} \frac{t^{n}}{n !}=\sum_{k=0}^{\infty} \frac{-t^{k}}{k !} \sum_{n=0}^{N-1} \frac{t^{n}}{n !}=1-\frac{t^{N}}{N !}+o\left(t^{N}\right)
$$

Re-write the cdf of $\mathrm{X}$ as:

$$
F_{w_{S R}}(x)=\frac{1}{L ! \bar{w}_{S R}^{L}} \cdot x^{L}+o\left(x^{L}\right)
$$

where $L=N \cdot Q$.

By substituting (B.2) and (3.17) into (3.16) with some algebraic manipulation, the cdf of wq as: 


$$
F_{w_{i}}(\gamma)=\underbrace{\int_{\gamma}^{\infty} \frac{y^{L-1}}{(L-1) ! \bar{w}_{R_{i}}^{L}} \cdot d y}_{a_{1}}-\underbrace{\int_{\gamma}^{\infty} \frac{(y+\gamma)^{L} y^{L-1}}{L !(L-1) ! \bar{w}_{S_{R}}^{L} \bar{w}_{R_{i}}^{L}} \cdot e^{\frac{y}{\overline{\bar{w}}_{R_{i}}}} \cdot d y}_{a_{2}} \text { (A.3) }
$$

Solving a1 by the identities in $8,3.381 .3]$ and [8, 8.352.4], the expression is obtained

$$
1-\frac{\gamma^{L}}{\bar{w}_{R_{i} D}^{L} L !}
$$

A2 is solved with the help of the generalized power series expansion for the exponential function in $[8,1.211 .1]$ and for $\Gamma(n ; t)$, the first order taylor series expansion is obtained as in:

$$
1-\frac{\gamma^{L}}{\bar{w}_{S_{i} R}^{L} L !(L-1) !}
$$

Substituting (A.5) in (19) using the integral in (20), then applying the identity in (A.1) after some algebraic manipulation, yields to the expression in (38).

\section{REFERENCES}

[1] Q. Li, Y. Yang, W. K. Ma, M. Lin, J. Ge and J. Lin. Robust Cooperative Beamforming and Artificial Noise Design for Physical-Layer Secrecy in AF Multi-Antenna Multi-Relay Networks. IEEE Transactions on Signal Processing, 2015 63( 1): 206-220.

[2] Kyeong Jin Kim and T. A. Tsiftsis. On the performance of cyclic prefix-based single-carrier cooperative diversity systems with best relay selection. IEEE Transactions on Wireless Communications, 2011, 10(4).

[3] A. Almradi and K. A. Hamdi,. Ergodic Capacity Analysis of MIMO Full Duplex Relaying with Imperfect CSI. IEEE Conference on Global Communications GLOBECOM, 2015: 1-6.

[4] T. Hesketh, R. C. de Lamare and S. Wales, Joint Partial Relay Selection, Power Allocation and Cooperative Maximum Likelihood Detection for MIMO Relay Systems with Limited Feedback. 77th IEEE Conference on Vehicular Technology(VTC), 2013: 1-5.

[5] C. Choudhuri and U. Mitra, "Rate bounds for relay channels using MIMO methods. 2010 44th Asilomar Conference on Signals, Systems and Computers, 2010: 1330-1334.

[6] Qiang Gao Jun Zhang, Li Fei and Xiao-Hong. Energyefficient multihop cooperative miso transmission with optimal hop distance in wireless ad hoc networks. IEEE Transactions on Wireless Communications, Oct 2011, 10(10): 3426-3435.
[7] C. C. Hu, G. F. Liu and B. H. Chen. Joint Relay/Antenna Selection and Precoding Design for Two-Way MIMO Amplify-and-Forward Relaying Systems. IEEE Transactions on Vehicular Technology 2016,, 65(7): 4854-4864.

[8] Liu, J., Shroff, N. B., \& Sherali, H. D. Optimal power allocation in multi-relay MIMO cooperative networks: Theory and algorithms. IEEE Journal on Selected Areas in Communications, 2012, 30(2): 331-340.

[9] M. Zhao, X. Gu, D. Wu and L. Ren. A two-stages relay selection and resource allocation joint method for $\mathrm{d} 2 \mathrm{~d}$ communication system. IEEE Conference on Wireless Communications and Networking. 2016: 1-6.

[10] Ha, H. K., Tuan, H. D., \& Nguyen, H. H. (2013). Joint optimization of source power allocation and cooperative beamforming for SC-FDMA multi-user multi-relay networks. IEEE Transactions on Communications, 2013,61(6):2248-2259.

[11] T. K. Y Lo, Maximum ratio transmission. IEEE Transactions on Communications, Oct 1990, 47(10): 1458,1461

[12] I. S. Gradshteyn and I. M. Ryzhik, Table of Integrals, Series, Products, New York NY, USA: Academic Press, seventh edition, 2007.

[13] I. B. Collings M. R. McKay, A. J. Grant, Performance analysis of mimomrc in double-correlated rayleigh environments. IEEE Transactions on Communications, 55(6):25-34.

[14] P. A. Anghel and M. Kaveh, Exact symbol error probability of a cooperative network in a Rayleighfading environment. IEEE Transactions on Wireless Communications, 2004, 3(9): 1416-1421.

[15] J. G. Proakis. Digital Communications. Prentice-Hall, fourth edition, 2011.

[16] I. A. stegun M. Abramowitz, Handbook of Mathematical Functions with Formulas, Graphs, Mathematical Tables, New York NY, USA: Dover, tenth edition, 1972.

[17] G. Lim and L. J. C. Jr, "Energy-efficient best-select relaying in wireless cooperative networks, IEEE Transactions on Communications, 2012, 6(3), 12-20.

[18] G. B. Giannakis Zhengdao Wang, A simple and general parameterization quantifying performance in fading channels. IEEE Transactions on Communications, 2003, 51(4).

[19] Crossbow Corporation. Mica2 datasheet [eb/ol]. 2008. www. eol. ucar. edu/rtf/facilities/isa/internal/CrossBow/ DataSheets/ mica2. pdf. 